\title{
La investigación social: tejiendo saberes desde voces plurales ${ }^{2}$
}

\author{
Social Research: \\ Weaving Knowledge from Multiple Voices
}

\begin{abstract}
Resumen
Reflexionar en torno a las prácticas profesionales es una tarea indispensable para el Departamento de Filosofía de Uniminuto. Este texto permite tener una aproximación a ello, - específicamente a la experiencia en investigación social llevada a cabo en el Centro de Estudios e Investigaciones Humanas y Sociales (CEIHS) De modo general, evidencia algunas consideraciones de la Filosofía junto con otros discursos en la investigación social, devela la importancia de la interdisciplinariedad, de la consciencia histórica y de la apuesta y compromiso político en dicho quehacer. El texto problematiza además el papel que juegan actualmente tanto la Filosofía como las Ciencias Humanas y Sociales en el contexto colombiano.
\end{abstract}

Palabras clave: Práctica profesional, investigación social, Filosofía, interdisciplinariedad, Ciencias Sociales, compromiso intelectual.

\begin{abstract}
To think about professional practices is a decisive task for philosophy department at Uniminuto. This paper leads to have an idea about it, specifically to the experience in social research carried out at Human and Social Research and Studies Center (CEIHSin Spanish). This document demonstrates some considerations of the philosophy in conjunction with other discourses in the social research; moreover, it unveils the importance of interdisciplinary, historical consciousness and the political commitment in this effort. Furthermore, the text problematizes the current role played by both philosophy and human and social studies in Colombian context.
\end{abstract}

Keywords: Professional Practice, Social Research, Philosophy, Interdisciplinary, Social Studies, Intellectual Commitment.

Recibido el 14 de mayo de 2013 y aprobado el 21 de mayo de 2013

1 Estudiante de último semestre de Estudios y Licenciatura en Filosofía de la Corporación Universitaria Minuto de Dios-Uniminuto. Correo electrónico: caroline.vargasg@gmail.com

2 Una primera versión de este texto fue presentada el día 3 de octubre de 2012 en el III Coloquio Interno de Prácticas Profesionales, en el marco del Encuentro Internacional Filosofía, Praxis y Sociedad realizado en Uniminuto. Es un artículo de reflexión. 
No podemos vivir eternamente/rodeados de muertos/y de muerte./

$Y$ si todavía quedan prejuicios / bay que destruirlos /"el deber"/ digo bien / EL DEBER/del escritor, del poeta, no es ir a/ encerrarse cobardemente en un texto,/ un libro, una revista de las que yal nunca más saldrá, sino al contrario/salir afuera / Para sacudir/para atacar / al espíritu público/si no / ¿Para qué sirve? / ¿Y para qué nació?

Antonin Artaud

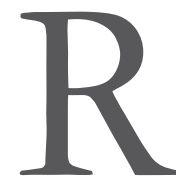
eflexionar y replantear los prejuicios que solemos tener es una de las cuestiones fundamentales en la tarea del tros supuestos a la hora de conocer e invest pueden no ser tan válidos como creemos es un aspecto fructífero al momento de construir el saber. El presente texto parte de estas consideraciones, del hecho mismo que es importante deconstruir nuestras experiencias teóricas y prácticas; es un primer intento argumentativo de la reflexión que he venido construyendo a lo largo de un poco más de un año y medio como practicante en el Centro de Estudios e Investigaciones Humanas y Sociales (CEIHS) de Uniminuto.

Desde el segundo semestre del año 2011 hasta el segundo de 2012 realicé mis tres prácticas profesionales en dos proyectos investigativos dirigidos por la docente-investigadora Angélica Nieto García ${ }^{3}$, proyectos que tenían la intencio- nalidad de indagar y reflexionar en torno a las memorias de las víctimas del Oriente antioqueño desde sus narrativas; el segundo proyecto estuvo centrado particularmente en el municipio de San Carlos, en el análisis de los efectos y consecuencias sociales que dejó la realización de la hidroeléctrica — construida durante las décadas de los setenta y ochenta en este municipio- y en las narrativas y memorias que construyen los habitantes alrededor de ello.

El objetivo central de la práctica era que, como estudiante de Filosofía, tuviera un acercamiento a la investigación social y desde mi formación académica aportara a los proyectos investigativos en los cuales participara. No obstante, mis expectativas iban más allá de ello, en tanto que ya venía replanteándome la necesidad de abrir otros caminos discursivos y prácticos que me permitieran comprender las problemáticas sociales que atraviesa el territorio colombiano; justamente esa fue una de las razones que me inclinaron a realizar mis prácticas en dicho campo.

El trabajo que realicé durante el primer semestre en el proyecto fue un acercamiento desde la tradición filosófica a la cuestión de la narrativa, ello básicamente desde el filósofo francés Paul Ricoeur. He de confesar que esto no fue fácil, pues durante dicha tarea consideré que acercarme a la problemática sólo desde este referente era muy limitado, más aún porque se trataba de entender un hecho social concreto, por ello fue necesario acudir a otros discursos como los de la Antropología y la Sociología para interpretar la problemática desde otras miradas y experiencias, sistematizadas alrededor de tal temática

En el segundo semestre, luego de algunas aproximaciones teóricas a la cuestión de la narración y la memoria, no muy claras por cierto, nos vimos en la necesidad de realizar un acercamiento al problema mismo que se estaba in-

3 A quien agradezco por permitirme trabajar a su lado conociendo y comprendiendo las dinámicas de la investigación social desde las difíciles problemáticas que envuelven la realidad histórica, política y social de Colombia; por posibilitarme el acercamiento al complejo tema de la memoria que me ha hecho comprender la importancia política del reconocer y construir junto a esas otra voces que ponen en cuestión la historia oficial. 
vestigando: la realidad social, política e histórica del Oriente antioqueño, de ahí que mi trabajo se centrara en la revisión de prensa del diario El Colombiano, concretamente de los archivos de los años 1997 y 1998; esta función la desarrollé en la hemeroteca de la Biblioteca del Banco de la República Luís Ángel Arango (BLAA), dicha revisión fue sistematizada en fichas. Además de ello, indagué en algunos documentos acerca de la historia y de las problemáticas del territorio.

Mi última práctica fue realizada en el segundo proyecto investigativo - por supuesto nada desligado del primero-, también en perspectiva de memoria, acerca del municipio de San Carlos, a propósito del impacto que ha tenido la construcción de la Central Hidroeléctrica y los discursos emergentes de la época en la cual fue construida; para ello continué realizando la revisión de prensa a El Colombiano, a la década del setenta, específicamente al año de 1978 y a algunas referencias de 1983, dicha búsqueda fue sistematizada para la investigación. Además de ello, fue necesario indagar y tomar como referencia la consideración de algunos trabajos e informes que se han realizado de San Carlos y del contexto del Oriente antioqueño.

Las páginas que aparecen a continuación pretenden, grosso modo, presentar algunas reflexiones que he construido gracias a mi experiencia como practicante. Cabe aclarar que si bien tomo algunas consideraciones de autores, el texto en su mayoría está construido desde apreciaciones subjetivas que se argumentan desde la experiencia misma.

\section{Reflexiones en torno a la práctica de la investigación}

Comprender es la facultad más propia de la existencia humana. Las diversas reflexiones inscritas a lo largo de la historia en todos los ámbitos humanos responden a ello, nacen de esa irremediable necesidad que nos acecha de encontrar sentidos a aquello que somos y a aquello que acontece en el territorio en el cual habitamos, de hallar horizontes a ese entramado de afecciones y pensamientos que acaecen entre lo ínfimo y lo abismal de la existencia. Es propio de nuestra condición humana comprender el presente donde interviene el pasado y se proyecta el futuro. Así, cada proyecto teórico corresponde, por más abstracto que parezca, a una comprensión desde la praxis de aquellos fenómenos y procesos fácticos que se nos presentan en ese entramado de hechos de la realidad.

Dejarnos afectar y reflexionar sobre aquello que nos circunda y que nos produce un sobresalto es tomar partida por la comprensión de la realidad dentro de nuestras posibilidades, de allí procede la tarea de investigar. Si bien es cierto que la búsqueda del conocimiento ha sido una constante en el devenir histórico, la noción de investigación tuvo una fervorosa acogida especialmente en la Edad Moderna por el arribo de la ciencia matematizada y cientificista.

La visión de la ciencia clásica, la cual aún predomina, se constituyó, en primer lugar, sobre las premisas del modelo de Newton, quien desde una visión teleológica expresaba que al igual que Dios, podemos alcanzar certezas, dado que todo coexiste en un eterno presente; y en segundo lugar, en el dualismo cartesiano, al suponer la distinción fundamental - perversa ruptura- entre la naturaleza material y física, y el mundo humano de lo social y la mente [...] Desde el siglo XVI se marca la diferencia entre ciencias naturales y ciencias sociales, dándoles a las primeras el carácter de un conocimiento cierto y a las segundas, el carácter de un conocimiento imaginado y aún imaginario (Molano, 2007, p. 281).

De ahí que, las Ciencias Sociales, en su búsqueda de una fundamentación propia de comprensión de lo social y lo humano asumieron, en un primer momento, en su quehacer los esquemas científicos ofrecidos por las Ciencias Naturales, las consideraciones positivistas también tuvieron un eco en ellas, cuestión que redujo las posibilidades de construir conocimiento desde miradas diversas, pues los principios de universalidad y adecuación, provenientes de la filosofía cartesiana y de la ciencia newtoniana, se tomaron como oficialización de aquello que 
efectivamente podía ser conocimiento verdadero. Por esta razón, entrado el siglo XX, las Ciencias Sociales se vieron en la necesidad de desligarse del modelo de adecuación científica y empezar a construir nuevos métodos y teorías para investigar

Las discusiones y debates epistemológicos a los cuales se enfrentaron las Ciencias Sociales por la influencia del naturalismo científico y el positivismo fueron influenciados y permeados al mismo tiempo por los acontecimientos que sucedieron en Europa entre los siglos XIX y XX; ello ayudó a fundamentarlas en tanto que se empezó a ver la necesidad de que las Ciencias Sociales posibilitaran pensar la realidad; enfrentaron y asumieron la tarea de plantear perspectivas que respondieran al nuevo orden social, buscando formas de pensamiento que acogieran rumbos para cimentar su identidad. En plena postguerra, las Ciencias Sociales estuvieron en crisis por la pertinencia requerida del contexto, tuvieron que redefinir y estructurar su función y finalidad, una propuesta que acogiera íntegramente lo humano, que fuese contextual e histórica y ayudara a transformar la realidad y a edificar nuevos sentidos de subjetividades, que por aquellos días estuvo tan precarizada y que relacionara además los saberes populares con lo científico para vincularlos con la cultura y el contexto social.

Desde esa misma línea, algunas filosofías occidentales del siglo XX adquieren —dado los hechos sociales, culturales y políticos de Europa- un carácter social y crítico que se desenvuelve no sólo a través de lo filosófico propiamente, sino a través de diversos discursos que se entrelazan para comprender el entramado de la realidad: la Filosofía no será el discurso por antonomasia sino uno más al servicio de dicha comprensión.

Pese a que hoy, gracias a la vertiginosa profesionalización y a los estereotipos y prejuicios generalizados, haya una lectura de la Filosofía como una disciplina o ciencia meramente teorética y abstracta que poco tiene que decir o aportar a los problemas de la crisis mundial que vivimos, es claro que tradicionalmente ésta ha sido un ejercicio de reflexión contextual que le viene apostando a incidir en las realidades sociopolíticas inmediatas, a partir de ella se ha pensado desde y para realidades particulares, se ha interpretado desde múltiples formas, contextos y problemas, que después de un tiempo se han avivado gracias a esas reflexiones.

Implicarse desde la perspectiva filosófica en una investigación de carácter social no es una tarea para nada sencilla, más aún cuando se guarda el fatídico presupuesto de que la Filosofía, al ser una reflexión que tiende generalmente a lo universal, puede brindar elementos para entender varios problemas. Intentar determinar cuál es el mejor camino para interpretar y aportar a la comprensión de un hecho social concreto, desde la tradición filosófica, puede ser desesperanzador cuando se encuentran varios puntos convergentes que luego, cuando existe un acercamiento más cerceno al hecho, dejan de ser suficientes para comprender el problema mismo. El fenómeno social desborda las teorías conceptuales desde las cuales se pretende tener una aproximación al problema.

Investigar lo social no es posible únicamente desde el discurso filosófico, la realidad y los hechos sociales que acaecen en ella son sumamente complejos y responden a tantos factores que se hace imposible comprenderlos desde una sola perspectiva. De ahí que considere que la investigación social, sea una tarea eminentemente interdisciplinar. La Filosofía se convierte dentro del conjunto de discursos en eso: en un discurso más que aporta elementos de comprensión. Contrario a lo que un par de veces he escuchado, afirmo que pensar lo social desde la Filosofía no quita la complejidad y rigurosidad a la reflexión filosófica; es aún mucho más compleja, rigurosa y enriquecedora, dado que se hace necesario no sólo comprender lo que dicen $\mathrm{X}$ o $\mathrm{Y}$ autores acerca de un concepto o problema, sino, por un lado, envolverse en otros discursos y en otras narrativas que recrean y ofrecen múltiples puntos de vista, y por otro, entender agudamente la realidad social, es decir, adentrarse en ella. Implica al mismo tiempo el 
ser y el hacer del investigador desde su subjetividad y desde la construcción intersubjetiva que se fortalece con los otros.

Para pensar la investigación social desde el aspecto interdisciplinar es necesario tener presente la mirada histórica y la comprensión de las particularidades contextuales del territorio que se está estudiando. La práctica investigativa abre el horizonte de interpretación de lo histórico como un pasado referido que es enclave del presente. La primacía histórica en la investigación social se debe a que en el campo de estudio de lo social se trata con contextos y subjetividades que han sido determinadas desde varios aspectos por lo histórico y que a la vez están forjándose y recreando la historia.

La conciencia y el rescate de la temporalidadespacialidad es una obligación manifiesta del investigador social que debe ir fortaleciendo no sólo por su tarea próxima - investigar-, sino desde el compromiso intelectual y social que tiene con su territorio, pues considero que todo quehacer intelectual o académico implica un ejercicio y compromiso de acción política. Las aproximaciones de comprensión a los hechos sociales exigen una penetración a partir de la historia; desde allí se vislumbra que lo histórico tiene cuantiosas implicaciones y que es conveniente acercarse a ello desde algunas interpretaciones, pues no se trata de afirmar sin más la historia oficial o de creer en una sola lectura. El comprender histórico devela la necesidad de acudir a diversas voces para penetrar en una mirada más completa.

Cabe hacer la cuña acá, a propósito de lo que se ha venido tratando, que desde mi experiencia en la práctica profesional he notado, con más agudeza, el llamado que tiene la Filosofía a rescatar los discursos narrativos, discursos que aportan un sinfín de elementos y que brindan lectu- ras múltiples desde las subjetividades. He podido entrever, además, que una de las caricaturizadas miradas que tenemos de la Filosofía como una disciplina infecunda y meramente libresca proviene del bache histórico que tenemos algunos de quienes hemos tenido un mediano contacto con la tradición filosófica, ello afirma aún más la posición de que las filosofías son reflexiones en el aire que no dependen ni están pensadas para un contexto específico.

La tarea pendiente que tenemos de comprender la historia (y cuando digo historia no me refiero a la noción de historia que se interpreta como objetiva, fija y progresiva, sino a una comprensión perspectivista —que implica múltiples puntos de vista-, compleja e incluso contradictoria de los hechos del pasado) es una necesidad manifiesta para los humanistas y científicos sociales. Considero que ese acercamiento a la conciencia histórica puede reflejar la tarea social que tienen y que deberían acoger de manera más comprometida las Ciencias Humanas y Sociales en Colombia.

Una de las consideraciones más sugestivas que aparecen al acercarse a la práctica investigativa es la implicación que el investigador empieza a tener con el problema y el hecho que estudia: su quehacer y la vida misma no son concebibles separados radicalmente. En otros términos, la investigación va caminando de la mano de la subjetividad del investigador, es un proceso constante donde las nociones de subjetividad, objetividad e intersubjetividad no son posibles separadamente, están involucradas al mismo tiempo. La objetividad tanto como la subjetividad dependen de lo intersubjetivo, el conocimiento se gesta desde diferentes voces, pues en el proceso de investigación social el encuentro con los otros y la realidad son el fundamento de comprensión, tanto de la objetividad que se le otorga al hecho estudiado como de la construcción dialógica de las subjetividades ${ }^{4}$.

4 A propósito de esto, considero que mi experiencia en la práctica hubiese sido indudablemente más fructífera si hubiera podido tener un acercamiento real al territorio y a los habitantes de San Carlos, por ejemplo; pues ello me permitiría hablar con propiedad desde la afección que esto podría haber causado. No obstante, considero lo anterior por las impresiones que me causó las experiencias que me relataba la investigadora Angélica Nieto a propósito de sus visitas al territorio. Sugiero que para próximas experiencias de estudiantes se piense en realizar trabajo de campo. 
La experiencia de quien investiga con sus encuentros fallidos y acertados es imprescindible en la tarea misma de la investigación. Muchas veces ingenuamente, en especial cuando se está "casado" con una manera teórica o metodológica de proceder, se tiende a pensar que investigar es un acto mecánico que conlleva a seguir pasos específicos —un poco por la mirada cientificista que aún tenemos de la investigación-, pero realmente no sucede así. Gracias a la hermenéutica gadameriana podemos afirmar acertadamente que la investigación no tiene un método ni una teoría trazada definitiva y que verdad y método no son dos aspectos intrínsecos, la verdad no se deriva sin más del método como consideró la Modernidad que equiparó la verdad al método científico. Existen múltiples sentidos que dependen de la interpretación mediada por la intencionalidad.

De la mano de los griegos y del sociólogo estadounidense Wright Mills podemos afirmar que la investigación es una artesanía, una techné y una praxis que se piensa a sí misma continuamente; una labor indescifrable que se desprende del proceso rígido y programado donde se cae en el fetiche del método y de la teoría. En los virajes diversos que toma la investigación social existe siempre el espacio de lo insospechado. El investigador se convierte, desde su multiplicidad de referentes teóricos y prácticos, en su propio maestro y aprendiz, de tal manera que un investigador social no puede pretender que una investigación proceda y sea igual a otra que ya ha construido, como quien hace algo mecánico, en dicha labor se presenta siempre la novedad.

La investigación social, al igual que las propias Ciencias Sociales, requiere de un constante fundamento teórico que esté vinculado con la praxis, desde allí se hace pertinente desmitificar e intentar desligarse de nociones filosóficas abstractas que se toman sin más como un fetiche. La cuestión anida en pensar los fenómenos y volver las reflexiones teóricas operativas en la realidad que se estudia, en forjar los conceptos, en teorizar y sintetizar a partir de la reflexividad social, allí reside precisamente la tarea de la Filosofía en la investigación social, con la par- ticularidad de que son los hechos mismos los que disciplinan y encaminan la reflexión y no las ideas y teorías las que deben proclamar cómo debe ser la realidad.

Así, la Filosofía, desde la investigación social, se enfrenta junto con otras disciplinas a rescatar los fenómenos socioculturales y a construir conocimiento contextualmente, se convierte en un discurso territorializado. La investigación requiere que en el territorio mismo en el cual se investiga se construyan formas de conocimiento, de ahí que el deconstruir conceptos y teorías ya predispuestas es también una tarea imprescindible. La labor fecunda consiste en plantear una dialéctica entre investigación empírica observante y conceptualización teórica.

Todos los discursos de una u otra manera responden, desde mi perspectiva, a la realidad desde la cual se piensan; las Ciencias Sociales y las Humanidades, entre ellas la Filosofía, tienen una función social de crítica, denuncia y resistencia al statu quo, si bien es cierto que su papel no es propiamente transformar, por lo menos debería tomarse en razón de su quehacer estas nociones, pues el hecho de pensar y afrontar los problemas sociales implica que se puedan hallar soluciones viables a ellos; implica que efectivamente la reflexión teórica debe intentar construir otros mundos, mundos posibles más vivibles. Lo anterior justifica el hecho de que es necesario que abandonen radicalmente el lugar burocrático que paradójicamente han tomado, pues el objetivo teleológico de analizar y comprender las estructuras sociales, además de penetrar en la comprensión de los hechos sociales, pretende develar las contradicciones y cuestiones que desean legitimarse como verdaderas, pero que guardan tras sí intereses específicos de poder; de ahí que los problemas a estudiar no deban responder directamente al orden y poder establecido. Como lo considera Wrigth Mills, el investigador en su oficio artesanal debe embestir al ethos burocrático, por ello es indispensable que quien se involucre en la investigación social deba relacionar sus inquietudes personales con las consideraciones a comprender públicamente. 
Sabed que el sentido humano de las cuestiones públicas debe revelarse relacionándolas con las inquietudes personales y con los problemas de la vida individual. Sabed que los problemas de la ciencia social, cuando se formulan adecuadamente, deben comprender inquietudes personales y cuestiones públicas, biografía e historia, y el ámbito de sus intrincadas relaciones (Wrigth Mills, 1986, p. 236).

Últimamente, gracias al debate coyuntural del papel del filósofo en la realidad colombiana que generó el artículo ¿Dónde están los filósofos?, publicado en la edición número 66 de la revista Arcadia, y las reacciones a él, e incluso algunas apreciaciones del texto Los profes de filosofía solo producen más profes de filosofía, publicado en la revista de la editorial de la Universidad Nacional de Colombia, han salido muchas críticas y defensas en torno al quehacer filosófico en el país, que la mayoría de veces está centralizado en la academia. Dichas críticas, que por supuesto tienen algo de razón, se centran en afirmar que la Filosofía es una disciplina abstracta y aislada que poco aporta a la reflexión de la realidad y que quienes se dedican a ello por profesión viven en "una torre de marfil", pues están distanciados del debate público y de lo que acontece en la vida nacional.

Estas críticas apreciaciones son, desde mi perspectiva, pertinentes no sólo para la Filosofía sino para las Ciencias Humanas y Sociales en general, pues a veces pereciese que el ejercicio interpretativo e investigativo desde estas disciplinas no responde más sino a un marco privado, a exigencias institucionales y a cuestiones burocráticas que develan que detrás de ello no existe un lugar de enunciación realmente crítico. Esta lamentable situación puede ser una consecuencia de la dominante profesionalización que hoy tienen estos saberes en el país y del mercado que se ha desarrollado en torno a ello.

No obstante, como lo enuncia Michel Foucault, el saber está unido al poder; de ahí que nuestra tarea como humanistas y como ciudadanos justamente está en buscar estrategias de poder que nos permitan accionar, desde las periferias y si es posible desde el centro, maneras de resistir, de incitación, que son necesarias para la construcción de la historia y que incluso pueden ser estrategias victoriosas de poder. Como lo indica el artículo de Arcadia, citando a Rubén Sierra: "el filósofo debe atender a su tiempo ¿No es hora ya de que los pensadores colombianos salgan de su fortín académico y entren decididamente en la discusión pública de los problemas del país?” (Restrepo, 2011). Ello por supuesto no es exclusivo para los filósofos, la exclamación recae para todos los pensadores colombianos, particularmente para los humanistas y científicos sociales.

Desde este sentido, considero que la investigación social es una tarea de acción política, pues el hecho de que analice y reflexione sobre territorios y subjetividades particulares implica necesariamente que no sea una labor estéril que enuncie juicios y teorías sin más acerca de cómo funciona la sociedad, sino que en su tarea de comprensión crítica manifieste juicios contundentes que apelen a transformar dicha realidad, de ahí que este quehacer no pueda tener bajo ninguna circunstancia una visión neutral como muchas veces se cree y pretende; debe enunciar un sentido teleológico enmarcado desde la política.

Las críticas y caminos que se plantean a los problemas sociales no pueden residir únicamente en un mero hacer intelectual que se estanque en la razón privada de quien investiga; deben proyectar una incidencia pública. Si a partir de la lectura de los discursos de las Humanidades y las Ciencias Sociales, y de la interpretación de la realidad somos conscientes de los diversos problemas estructurales que enfrenta, por ejemplo, Colombia, ¿por qué no decirlo?, ¿por qué quedarse callado ante ello?, ¿por qué no rescatar esas otras miradas, de las cuales reconocemos que también enuncian algo de la realidad y contradicen esa historia oficial que muchas veces desde la academia se ayuda a oficializar?

La Filosofía indaga acerca de la condición humana. El hecho de que su quehacer resida en comprender y conceptualizar el mundo implica que la tarea de ésta, desde la investigación social interdisciplinar, consista en pensar la realidad 
que involucra lo histórico, lo social, lo político, lo económico etc., bajo una misma esfera; en desenmascarar y hacer evidente lo no manifiesto, en deconstruir métodos y teorías que permitan comprender el entorno cambiante y contradictorio en el cual habitamos; en hacer del conocimiento un constructo regional e intersubjetivo. Una de las enseñanzas personales más importantes que he podido vislumbrar en esta reflexión del quehacer filosófico en la investigación social es que, en definitiva, necesitamos menos mapas preestablecidos y más territorios: más apertura a caminos investigativos desde lo inadvertido y menos programación desde el fetichismo conceptual y teórico, más apertura a interpretar nuestra vida privada y pública y menos apego a los cartogramas de la tradición, que si bien son indispensable conocerlos, no nos basta con ello.

Mi experiencia en investigación social como estudiante de Filosofía ha sido bastante gratificante, académica y personalmente, sobre todo por los encuentros un tanto "fallidos" desde la tradición filosófica que he tenido para comprender un problema tan real y latente como lo es el de las memorias de las víctimas en el conflicto colombiano, que si bien aporta muchos elementos de base para entenderlo, la problemática desborda los intentos de aproximación filosófica; por ello, la interdisciplinariedad, la eminente importancia de la conciencia histórica y la acción política de crítica y denuncia, que mencioné líneas arriba, son los elementos más significativos que he acogido como base desde la experiencia.

Considero que si bien el quehacer filosófico debe continuar con su tarea rigurosa de interpretación a la tradición, algunas filosofías deben abrir caminos de comprensión a las problemáticas actuales que vivimos quienes estamos involucrados en este proceso; deben tomar otros discursos que les aportan elementos en esa tarea de comprensión. No obstante, dicha tarea es algo que queda abierto, no a la tradición filosófica propiamente sino a quienes hemos tenido algún acercamiento a ella y que somos concientes de que el discurso filosófico, junto con el de las Humanidades y las Ciencias Sociales, tienen mucho que hacer y aportar a la construcción humana, especialmente en un país como Colombia, donde resulta imposible no dejarse afectar e implicar de una u otra manera en las situaciones que han venido aconteciendo por más de sesenta años y que siguen prolongando injusticias y crímenes, en general en Latinoamérica, donde nuestra historia sigue siendo una lucha de resistencia.

A pesar de las diferentes apuestas que nos han dejado algunos filósofos de la tradición, recientemente los intelectuales occidentales del siglo XX como Sartre, Hannah Arendt, Horkeheimer, Foucault, Deleuze, y algunos latinoamericanos como Enrique Dussel, Paulo Freire, Estanislao Zuleta y Guillermo Hoyos, de que la tarea de quien se encamina en el auténtico filosofar es interpretar la realidad e intentar una metamorfosis, existen aún muchos personajes: los pseudointelectuales, que hoy se autoproclaman filósofos porque "saben" e interpretan la tradición occidental. Si la realidad colombiana no les dice nada a ellos y a nosotros tampoco, creo que en realidad estaríamos en un camino libresco e infecundo, pero soy totalmente consciente de que no es así. Colombia y en general Latinoamérica son libros siempre abiertos que están a la espera de ser conocidos, releídos y recreados desde otras voces y miradas de empoderamiento.

Por último, quisiera referirme a algo particular que nos compete a quienes somos parte del Departamento de Filosofía de Uniminuto, a propósito de la proyección social que intentan justificar y extender desde el quehacer filosófico; he de considerar algunas aspectos: desde mi experiencia y formación académica, encuentro, ahora que tengo una visión un poco más amplia de cómo se investiga socialmente, falencias formativas en investigación social, desde el programa son muy pocas las herramientas y elementos investigativos que he encontrado para realizar una investigación social. De hecho, también han sido muy pocas las veces que hemos realizado una reflexión o análisis de un problema social concreto desde la tradición 
filosófica. Más aún, parece paradójico pero he notado en el encuentro con mis compañeros que hay un problema generalizado de no saber proceder en el estudio de un hecho social, de no tener elementos esenciales para formular e intervenir en una investigación no sólo de carácter práctico sino también teórico; cuestión que es preocupante porque ello trunca muchas de las perspectivas personales y generales de la investigación en Filosofía que ha venido construyendo el Departamento.

A pesar de ello, paradójicamente esto ha sido para mí, desde la experiencia en la práctica profesional algo interesante, dado que me he visto en la tarea de realizar una lectura e investigación en diversos discursos y fuentes. Sin embargo, no deja de ser alarmante el hecho de que se suponga que estamos preparados para una labor en la cual en realidad no tenemos bases bien fortalecidas. Pese a las falencias, considero que continuar con el campo de práctica en investigación

\section{Referencias bibliográficas}

Artaud, A. (1967). Carta a los poderes. Buenos Aires: Insurrexit.

Mills, W. (1986). La imaginación Sociológica. México D.F.: F. C. E.

Molano, J. (2007). Las Ciencias Sociales como integración de saberes en la dimensión humana. Tabula rasa, 7, 275-303. social es importante para fortalecer estos aspectos. Además de ello creo que sería pertinente extender la apertura de cursos y seminarios que posibiliten el acercamiento a reflexiones de problemas sociales y a técnicas y herramientas de investigación social.

Decido poner este aspecto en consideración porque pienso que es necesario que se cuestionen las prácticas académicas e investigativas por parte de estudiantes y docentes, que se reconozca en qué estamos fallando, qué falencias tenemos y cómo podemos superarlas en conjunto; replantear cuáles son nuestras prioridades, alcances, fortalezas y debilidades. Cabe preguntarnos justamente acá si la labor que venimos realizando sigue cayendo en las prácticas librescas y frívolas que hemos criticado durante un buen tiempo o si aquello que estamos haciendo, realmente, puede dar frutos en términos de una apuesta social desde la comprensión filosófica.
Palacio, R. (abril, 2012). Los profes de filosofía solo producen más profes de filosofía. $L a$ revista editoriUNal, s/n, 18.

Restrepo, R. (marzo 24, 2011). ¿Dónde están los filósofos? Arcadia, 66. Recuperado de: http://www.revistaarcadia.com/impresa/ filosofia/articulo/donde-estan-filosofos $/ 24577$ 